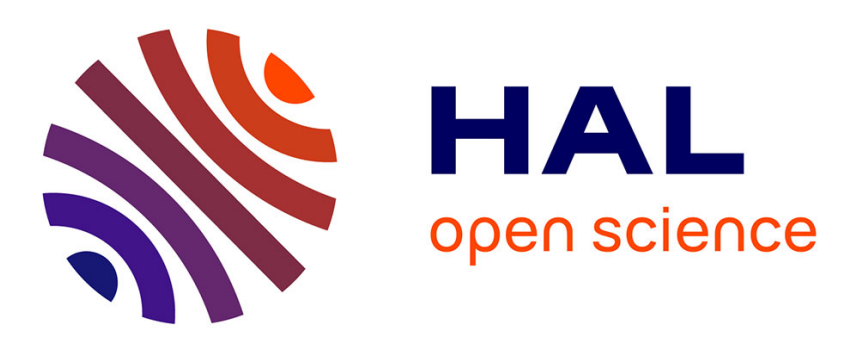

\title{
Détermination de la réponse asymptotique d'une structure anélastique sous chargement thermomécanique cyclique
}

Habibou Maitournam, Benjamin Pommier, Jean-Jacques Thomas

\section{- To cite this version:}

Habibou Maitournam, Benjamin Pommier, Jean-Jacques Thomas. Détermination de la réponse asymptotique d'une structure anélastique sous chargement thermomécanique cyclique. Comptes Rendus Mécanique, 2002, 330, pp.703-708. 10.1016/S1631-0721(02)01516-4 hal-00111359

\section{HAL Id: hal-00111359 \\ https://hal.science/hal-00111359}

Submitted on 15 Jul 2019

HAL is a multi-disciplinary open access archive for the deposit and dissemination of scientific research documents, whether they are published or not. The documents may come from teaching and research institutions in France or abroad, or from public or private research centers.
L'archive ouverte pluridisciplinaire HAL, est destinée au dépôt et à la diffusion de documents scientifiques de niveau recherche, publiés ou non, émanant des établissements d'enseignement et de recherche français ou étrangers, des laboratoires publics ou privés. 


\title{
Détermination de la réponse asymptotique d'une structure anélastique sous chargement thermomécanique cyclique
}

\author{
M. Habibou Maitournam a , Benjamin Pommier a,b, Jean-Jacques Thomas b \\ a Laboratoire de mécanique des solides (CNRS UMR 7649), École polytechnique, 91128 Palaiseau, France \\ b PSA-DRIA/SARA/MATE, chemin de la Malmaison, 91570 Bièvres, France
}

Résumé Cet article présente une solution alternative aux méthodes classiques (comme la méthode incrémentale) de résolution de problèmes thermomécaniques cycliques non-linéaires. Il s'agit d'une Méthode Cyclique Directe qui consiste à rechercher directement la solution asymptotique d'une structure anélastique soumise à un chargement thermomécanique périodique, sans suivre l'histoire du chargement. Elle est fondée sur le Grand Incrément de Temps et la périodicité de l'état limite et la transformation de Fourier.

solides et structures / cycle limite / thermomécanique / méthode numérique

\section{Determination of the asymptotic response of a structure under cyclic thermomechanical loading}

Abstract A method for the solution of cyclic thermomechanical nonlinear problems is presented: it is a Direct Cyclic Method which is an alternative approach to classical methods. It consists in directly seeking the asymptotic response of an inelastic structure subjected to a periodic thermomechanical loading without following the loading path. It is based on the Large Time Increment Method, the periodicity of the limit state and the Fourier transform.

solids and structures / limit cycle / thermomechanics / numerical method

\section{Abridged English version}

The prediction of the lifetime of inelastic structures under cyclic thermomechanical loading requires, on the one hand, the following of the response evolution until a possible stabilized state (which could be obtained directly) is reached, and on the other hand, the use of an appropriate damage criterion. The computation of the whole loading history leads to lenghty and expensive incremental calculations, especially for structures with large number of degrees of freedom. Alternative strategies to the step by step method have been proposed [2-4].

Adresses e-mail : habibou@lms.polytechnique.fr (M.H. Maitournam); benjamin.pommier@mpsa.com (B. Pommier); jean-jacques.thomas@mpsa.com (J.-J. Thomas). 
In a design approach recently developed [1] for an automotive engine undergoing thermal fatigue, only the periodic limit response is interesting. The aim of this paper is to develop a Direct Cyclic Method for the direct determination of the limit cycle of an inelastic structure subjected to thermomechanical cyclic loading. It derived from the Large Time Increment Method [2] and the direct method developed by Akel and Nguyen [5], and uses Fourier expansion in solving the global equations.

More precisely, we deal with an inelastic structure subjected to a cyclic loading. The strategy adopted use the Fourier transform in time, as proposed by Cardona et al. [7], to calculate the solution over the whole stabilized cycle $[0, T]$. At each time $t_{j}$ of the discretized cycle $[0, T]$, the virtual power principle leads to Eq. (1), which Fourier expansion is given by (2). The satisfaction of the weak form of this Eq. (3) is then sought. It leads to the solution of the system (4) in which the tangent matrix is given by (7), or (8) when a quasi-Newton method is used. The numerical procedure effectively used for the determination of the asymptotic response consists in: (i) a global stage: where statically and kinematically admissible (SKA) solutions with the given forces and displacements are determined for the whole cycle; (ii) a local stage: where plastically admissible (PA) solutions for the whole cycle, are derived from the (SKA) solutions; a radial return algorithm [8,9] in used; (iii) periodicity: the periodicity condition is prescribed to all mechanical quantities. The process stops when the difference between the periodic mechanical SKA quantities and the PA ones is small. In the case of ratchetting, the method does not converge.

The global stage is linear over the entire cycle: we use Fourier transform with the constant stiffness (8).

An illustration of this method is given on the example of a structure which is representative of the part of a cylinder head, most sensitive thermomechanical fatigue. The material used is elastoviscoplastic [10]. The structure is subjected to a thermal loading with strong gradient. The temperature cycle at the most severely loaded point is given in Fig. 1. In Fig. 2 a comparison of the stabilized cycles at this point, obtained by the Direct Cyclic Method and the step by step method is shown. Fig. 3 shows the contours of a component of the plastic strain obtained by the two methods: a good agreement is found.

\section{Introduction}

La prédiction de la durée de vie de structures anélastiques soumises à des chargements thermomécaniques nécessite le suivi de l'évolution thermomécanique jusqu'à un éventuel état stabilisé (ou sa recherche directe) et l'utilisation de critères d'endommagement appropriés. Pour de telles structures soumises à des chargements thermomécaniques cycliques (comme les culasses automobiles), une approche globale de dimensionnement des structures a été récemment développée [1]. Elle s'articule autour de quatre points principaux suivants : (i) définition d'un chargement thermomécanique représentatif; (ii) modélisation représentative du comportement du matériau dans la pièce (loi de comportement élastoviscoplastique avec découplage de l'endommagement); (iii) résolution numérique (détermination du cycle stabilisé de la structure); (iv) critère prédictif de durée de vie à partir de l'énergie mécanique dissipée pendant le cycle stabilisé.

Cette démarche est actuellement utilisée en bureau d'études pour dimensionner certaines structures comme les collecteurs d'échappements. Cependant les techniques de résolutions numériques utilisées (méthode incrémentale) conduisent à des temps de calcul trop longs pour déterminer la réponse cyclique des structures à grand nombre de degrés de liberté $\left(10^{5}\right.$ à $\left.10^{6} \mathrm{ddl}\right)$. Il est donc nécessaire de développer des méthodes numériques alternatives afin de rendre ces temps de calcul compatibles avec les exigences des bureaux d'études. Plusieurs techniques ont déja été proposées. La méthode à grand incrément de temps [2] utilise une stratégie fondée sur l'utilisation d'une double échelle de temps pour représenter les évolutions rapides sur un cycle et lentes entre les cycles. D'autres méthodes se proposent de rechercher directement l'éventuel cycle stabilisé ; la plus récente est fondée sur l'utilisation de l'approche du contrôle optimal [3]. La méthode simplifiée proposée par Zarka [4] se base sur une estimation des paramètres internes de l'état stabilisé, à partir de la réponse cyclique purement élastique. 
Cet article porte sur le développement d'un algorithme, qui permet, par un processus itératif, de déterminer directement la réponse asymptotique de la structure, sans calculer les cycles transitoires pendant lesquels la structure s'adapte ou s'accommode. On illustrera cette méthode par la simulation d'une structure simplifiée représentative d'une partie chaude de culasse et qui sera comparée à une simulation par une méthode incrémentale.

\section{Position du problème}

Nous nous intéressons à un solide de volume $\Omega$ soumis au chargement cyclique de période T suivant : efforts surfaciques $\boldsymbol{F}(\boldsymbol{x}, t)$ appliqués sur la frontière $\partial_{\boldsymbol{F}} \Omega$, efforts de volume $\boldsymbol{f}(\boldsymbol{x}, t)$ appliqués dans $\Omega$ et déplacements imposés $\boldsymbol{U}(\boldsymbol{x}, t)$ sur la frontière $\partial_{U} \Omega$. De plus, il est soumis en tout point à un champ de température T-périodique issu d'un calcul découplé (on néglige l'influence de la dissipation mécanique et du couplage thermoélastique sur la réponse thermique). Son évolution est supposée quasi-statique en transformations infinitésimales. Le comportement du matériau considéré est élastoviscoplastique standard généralisé.

Nous cherchons la réponse périodique $(\boldsymbol{\sigma}(\boldsymbol{x}, t), \boldsymbol{u}(\boldsymbol{x}, t))$ en tout point $x$ du solide $\Omega$ à chaque instant $t$ du cycle stabilisé - lorsque celui-ci existe - noté $[0, T]$. Celle-ci est constituée, à tout instant, d'un champ de contraintes $\boldsymbol{\sigma}(\boldsymbol{x}, t)$ statiquement admissible (SA) avec les données en efforts du problème et d'un champ de déplacement $\boldsymbol{u}(\boldsymbol{x}, t)$ cinématiquement admissible (CA) avec les données en déplacement du problème reliés par la loi de comportement élastoviscoplastique.

\section{Principe de la méthode}

Les structures soumises à des chargements périodiques peuvent présenter les différents types de comportement asymptotique (lorsque le temps $t$ tend vers l'infini) suivants : l'adaptation (la structure peut subir des déformations inélastiques durant les premiers cycles, mais sa réponse asymptotique est élastique et l'énergie dissipée reste bornée), l'accommodation (la structure tend vers un cycle inélastique stabilisé, c'està-dire que les déformations inélastiques et les variables internes évoluent à chaque cycle mais retrouvent leurs valeurs initiales à la fin de chaque cycle), le rochet (les déformations inélastiques s'accumulent à chaque cycle).

Dans notre approche de dimensionnement, où l'on suppose que le nombre de cycles nécessaires à l'obtention d'un état limite est négligeable devant le nombre de cycles à la rupture, seule la réponse asymptotique est intéressante pour déterminer la tenue à la fatigue de la structure. C'est pourquoi on se propose de la rechercher directement, sans calculer les cycles préliminaires.

Dans son principe, la démarche s'appuie sur la méthode à grand incrément de temps développée par Ladevèze et al. [2] et la recherche directe de cycle stabilisé proposée par Akel et Nguyen [5] et dans [6]. De ces travaux on retient les deux principes fondamentaux suivants pour la méthode cyclique :

Séparation des étapes globales et locales : La difficulté de résolution du problème non-linéaire vient de la coexistence d'équations globales en espace (c'est-à-dire nécessitant l'inversion d'un système à grand nombre de degré de libertés) et d'équations locales, non-linéaires, de comportement. On va donc séparer le traitement de ces étapes sur l'ensemble du cycle.

Recherche de solutions périodiques : Les solutions recherchées sont périodiques. On va donc imposer cette condition dans le schéma itératif de résolution.

\section{Résolution générale du problème non-linéaire}

Le principe des puissances virtuelles nous donne : $\forall t \in[0, T], \forall \boldsymbol{u}_{t}^{*}(\boldsymbol{x}) \mathrm{CA}$ à 0 à l'instant $t$,

$$
r(\boldsymbol{u}(\cdot, t))=\int_{\Omega} \boldsymbol{\sigma}(\boldsymbol{u}, t): \boldsymbol{\varepsilon}\left(\boldsymbol{u}_{t}^{*}\right) \mathrm{d} \Omega-\int_{\Omega} \boldsymbol{f}(\boldsymbol{x}, t) \cdot \boldsymbol{u}_{t}^{*}(\boldsymbol{x}) \mathrm{d} \Omega-\int_{\partial_{F} \Omega} \boldsymbol{F}(\boldsymbol{x}, t) \cdot \boldsymbol{u}_{t}^{*}(\boldsymbol{x}) \mathrm{d} S=0
$$


On discrétise l'intervalle d'étude $[0, T]$ en $N$ instants notés $t_{j}$. Cette équation (1) est valable à chaque instant $t_{j}$. Elle est non-linéaire en $\boldsymbol{u}$ et peut se décomposer en séries de Fourier sous la forme :

$$
r_{j}=r\left(\boldsymbol{u}\left(t_{j}\right)\right)=\frac{R_{0}}{2}+\sum_{m=1}^{N-1} R_{m}^{c} \cos \left(2 \pi m \frac{t_{j}}{T}\right)+R_{m}^{s} \sin \left(2 \pi m \frac{t_{j}}{T}\right)
$$

La recherche de la solution s'inspire de [7]. Au lieu de rechercher les solutions $\boldsymbol{u}_{j}=\boldsymbol{u}\left(t_{j}\right)$ qui annulent le résidu $r_{j}$ (2), on va rechercher les solutions qui annulent les différents coefficients de Fourier $R_{m}^{c / s}$.

$$
R_{m}^{c}=\frac{2}{T} \sum_{j=0}^{N-1} r_{j} \cos \left(2 m \pi \frac{t_{j}}{T}\right)=0 \quad \text { et } \quad R_{m}^{s}=\frac{2}{T} \sum_{j=0}^{N-1} r_{j} \sin \left(2 m \pi \frac{t_{j}}{T}\right)=0
$$

Ces équations sont non-linéaires en $\boldsymbol{u}$ (car $r_{j}$ l'est). On va les résoudre par un schéma de type NewtonRaphson. La solution peut donc être approchée par le processus itératif suivant :

- étape globale :

$$
\left(U_{n}^{c / s}\right)^{i+1}=\left(U_{n}^{c / s}\right)^{i}+\left(\Delta U_{n}^{c / s}\right)^{i+1} \quad \operatorname{avec}\left(\Delta U_{n}^{c / s}\right)^{i+1}=-\left(\frac{\partial R_{m}^{c / s}}{\partial U_{n}^{c / s}}\right)^{-1}\left(R_{m}^{c / s}\right)^{i}
$$

- étape locale : calcul de $\left(R_{m}^{c / s}\right)^{i+1}$.

Dans le cadre de ce processus itératif, la difficulté réside dans l'écriture de la matrice tangente. A titre d'illustration on va expliciter le terme $\partial R_{m}^{c} / \partial U_{n}^{c}$. Le terme $R_{m}^{c}$ peut s'écrire :

$$
R_{m}^{c}=\frac{2}{T} \sum_{j=0}^{N-1} r\left(\frac{U_{0}}{2}+\sum_{n=1}^{N-1} U_{n}^{c} \cos \left(2 \pi n \frac{t_{j}}{T}\right)+U_{n}^{s} \sin \left(2 \pi n \frac{t_{j}}{T}\right)\right) \cos \left(2 m \pi \frac{t_{j}}{T}\right)
$$

En dérivant par $U_{n}^{c}$ on a :

$$
\frac{\partial R_{m}^{c}}{\partial U_{n}^{c}}=\frac{1}{T} \sum_{j=0}^{N-1} \frac{\partial r}{\partial u}\left(u_{j}\right)\left(\cos \left(2 \pi(n+m) \frac{t_{j}}{T}\right)+\cos \left(2 \pi(n-m) \frac{t_{j}}{T}\right)\right)=K_{n+m}^{c}+K_{n-m}^{c}
$$

On note $k_{j}=\frac{\partial r}{\partial u}\left(u_{j}\right)$, la matrice tangente à l'instant $t_{j}$ et $K_{n}^{c / s}$ le coefficient du développement en série de Fourier de la matrice $k(t)$. En procédant de même pour les autres termes, on obtient la matrice suivante :

$$
\left[\frac{\partial R_{m}^{c / s}}{\partial U_{n}^{c / s}}\right]=\left[\begin{array}{ccccc}
K_{0}^{c} & \cdots & K_{n}^{c} & K_{n}^{s} & \cdots \\
\vdots & & \vdots & \vdots & \\
K_{m}^{c} & \cdots & \left(K_{n+m}^{c}+K_{n-m}^{c}\right) & \left(K_{n+m}^{s}-K_{n-m}^{s}\right) & \cdots \\
K_{m}^{s} & \cdots & \left(K_{n+m}^{s}+K_{n-m}^{s}\right) & \left(K_{n-m}^{c}-K_{n+m}^{c}\right) & \cdots \\
\vdots & & \vdots & \vdots
\end{array}\right]
$$

Cette matrice est de taille $N$ fois le nombre de ddl. Pour se ramener à une résolution plus simple, on utilise un schéma de type quasi-Newton en choisissant la matrice $k_{j}$ constante au cours du temps et égale à sa valeur initiale. La matrice (7) s'écrit alors :

$$
\left[\frac{\partial R_{m}^{c / s}}{\partial U_{n}^{c / s}}\right]=\left[\begin{array}{llll}
K & & & 0 \\
& K & & \\
& & \ddots & \\
0 & & & K
\end{array}\right]
$$

$\mathrm{Si}$ on tronque les développements en série de Fourier à un nombre de termes suffisant pour avoir une bonne approximation mais assez petit, on diminue le nombre d'équations d'équilibre à résoudre par rapport à un calcul incrémental classique. La solution temporelle en déplacements et en déformations sur l'ensemble du cycle est obtenue par recomposition à partir des coefficients de Fourier $U_{n}$. 


\section{Résolution pratique}

Le processus itératif de la méthode cyclique directe se décompose alors en trois étapes.

- Etape globale : on recherche des solutions statiquement et cinématiquement admissibles (SCA) avec les forces et les déplacements donnés, à chaque instant du cycle; Pour cela, on utilise la matrice (8), en tronquant les séries de Fourier pour diminuer le nombre de résolutions mais en gardant suffisamment de termes pour ne pas trop détériorer la solution.

- Etape locale : on détermine des solutions plastiquement admissibles sur tout le cycle, à partir des solutions SCA, de manière implicite, par projection orthogonale de celles-ci sur le convexe de plasticité (algorithme de retour radial) [8,9] entre deux instants consécutifs.

- Périodicité : on impose la condition de périodicité des grandeurs mécaniques et des variables internes. La différence par rapport à Akel et Nguyen [5] réside essentiellement dans le fait qu'ils recherchent par itérations, lors de l'étape locale, une solution périodique plastiquement admissible alors qu'ici la condition de périodicité est imposée.

L'arrêt du processus est déterminé par la périodicité des grandeurs mécaniques SCA et leur admissibilité plastique. En cas de rochet, la méthode ne converge pas.

\section{Résultats numériques}

Pour illustrer cette méthode, nous présentons l'exemple d'une structure ayant une géométrie et un chargement thermique représentatif de la zone de la culasse la plus sensible à la fatigue thermomécanique. Le modèle de comportement utilisé pour simuler le matériau est un modèle élastoviscoplastique dont les détails de l'implémentation numériques sont données dans [10]. Cette pièce est soumise à un chargement thermique cyclique à fort gradient. Le cycle de température au point le plus chargé est représenté sur la Fig. 1. Sur la Fig. 2, on peut comparer le cycle stabilisé obtenu au point le plus chargé par la méthode incrémentale où la stabilisation est obtenue après une quarantaine de cycles, et celui obtenu par la méthode cyclique directe. La Fig. 3 montre la bonne corrélation entre les champs de déformations inélastiques obtenus par le calcul incrémental et par la méthode cyclique directe.

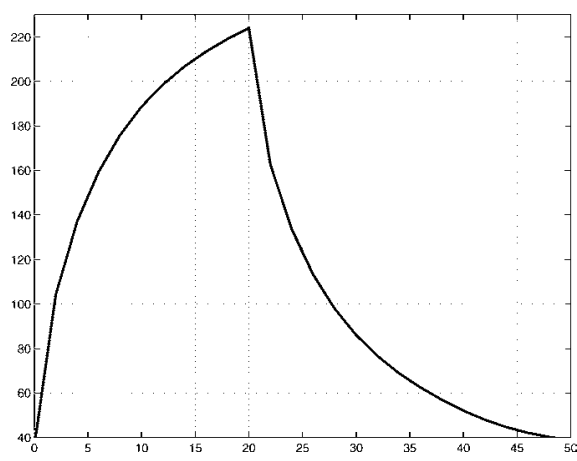

Figure 1. Cycle de température imposé.

Figure 1. Cycle of the prescribed temperature.

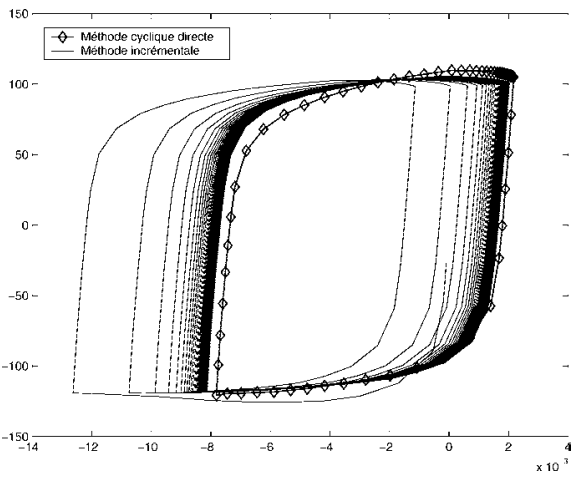

Figure 2. Cycle contrainte-déformation obtenu par la méthode cyclique directe et par un calcul incrémental.

Figure 2. Stress-strain cycle obtained by the Direct Cyclic Method and the incremental analysis. 

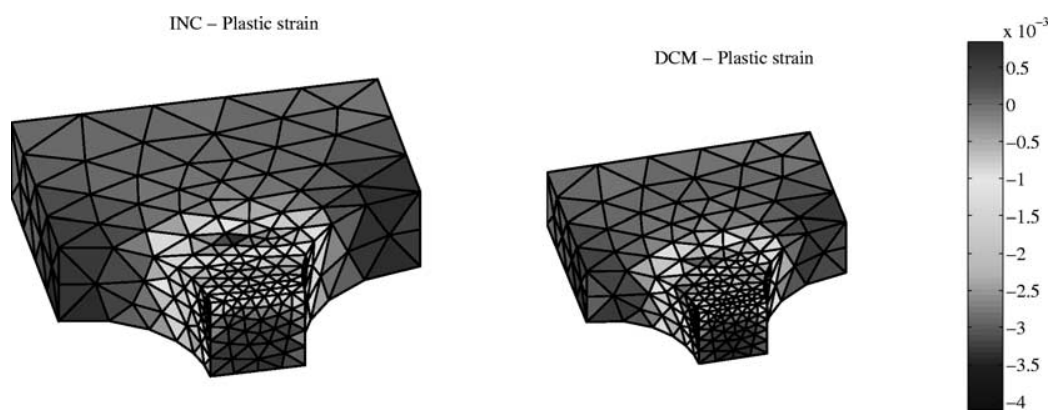

Figure 3. Déformation plastique à la fin du cycle stabilisé obtenue par un calcul incrémental et par la méthode cyclique directe.

Figure 3. Plastic strain at the end of the stabilized cycle obtained by the Direct Cyclic Method and the incremental analysis.

\section{Conclusion}

La méthode cyclique directe permet de déterminer le comportement asymptotique d'une structure anélastique soumise à un chargement thermomécanique cyclique. L'état stabilisé (adaptation ou accommodation) est obtenu par un algorithme qui s'inspire de différents travaux, mais qui est particulièrement original par sa résolution de l'équilibre de la structure dans l'espace fréquentiel. Cela permet de diminuer le temps de calcul, particulièrement dans le cas des structures à grand nombre de degrés de liberté. La faisabilité de l'utilisation de cette méthode dans le cas de simulations de structures anisothermes fortement non-linéaires est démontrée.

\section{Références bibliographiques}

[1] E. Charkaluk, A. Constantinescu, A. Bignonnet, K. Dang Van, Dimensionnement des structures à la fatigue thermique, in : 18ème journées de printemps de la SF2M, Paris, 12(1)-12(8), 1999.

[2] P. Ladeveze, Mécanique non linéaires des structures, Hermès, Paris, 1996.

[3] M. Peigney, C. Stolz, Approche par contrôle optimal des structures élastoviscoplastiques sous chargement cyclique, C. R. Acad. Sci. Paris, Série IIb 329 (2001) 643-648.

[4] J. Zarka, J. Frelat, G. Inglebert, P. Kasmai-Navidi, A new approach in inelastic analysis of structures, CADLM, Gif-sur-Yvette, 1990.

[5] S. Akel, Q.S. Nguyen, Determination of the limit response in cyclic plasticity, in: D.R.J. Owen, E. Hinton, E. Onate (Eds.), Computational Plasticity: Models, Software and Applications, 1989, pp. 639-650.

[6] N. Maouche, M.H. Maitournam, K. Dang Van, On a new method of evaluation of the inelastic state due to moving contacts, Wear 203-204 (1997) 139-147.

[7] A. Cardona, T. Coune, A. Lerusse, M. Geradin, A multiharmonic method for non-linear vibration analysis, Int. J. Numer. Methods Engrg. 37 (1994) 1593-1608.

[8] Q.S. Nguyen, On the elastic plastic initial-value problem and its numerical integration, Int. J. Numer. Methods Engrg. 11 (1977) 817-832.

[9] J.C. Simo, T.J.R. Hughes, Computational Inelasticity, Springer-Verlag, New York, 1998.

[10] L. Verger, A. Constantinescu, E. Charkaluk, On the simulation of large viscoplastic structures under anisothermal cyclic loading, in: O. Murakami (Ed.), IUTAM Creep in Structures, Kluwer Academic, 2000. 